Model Behavior: Animal Experiments, complexity and the genetics of psychiatric disorders, by Nicole C. Nelson, Chicago and London: University of Chicago Press, 2018, 255 pp.,

\title{
Reuben Message
}

In Model Behavior, Nelson examines how animal behavior geneticists work to sustain the technical and discursive infrastructures that underpin the epistemic integrity of the mouse models they use, and consequently the order of knowledge their field produces. This work is neither easy nor ever complete. Drawing on extensive field interviews and ethnographic graft, primarily in the neuroscience department and labs of a university on the West Coast of the United States, she shows that the authority of the mouse as a knowledge-making tool is an achievement requiring continuous efforts to justify.

This work is needed because of the complexity of what is modelled and the social context in which this modelling takes place. Psychiatric disorders (Nelson concentrates on addiction behaviors) are intrinsically complicated. There are no simple means to gauge the likeness of mouse and human behaviour in this field, and the phenomena are also highly environmentally dependent. The number of variables to control escalates. The standardised model organism used is only a beginning; indeed, the environment itself calls for standardisation, and claims about model validity need to be judge in relation to environmental conditions. Moreover, the wider field of behavioral genetics itself is awash with social controversies, resulting in forms of scrutiny which make the job of maintaining model validity challenging. Complexity thus emerges as both an ontological property that researchers grapple with and, as Nelson emphasizes, an epistemological impediment, roughly equivalent to uncertainty (p. 50) that researchers use to understand and police knowledge claims in their field.

The book is admirably located in the literature. It builds on the theme of complexity that dominates postgenomic bioscience. It's defence of a constructionist outlook in science and technology studies (STS), vis-à-vis alternative trends (notably the new materialisms) is particularly well handled. Returning to a primal scene for the discipline - the laboratory study - Nelson revisits the fundamental notion that the analysis of the production of scientific knowledge involves tracing the social processes through which the natural order emerges as objectively given fact. Her major contribution is showing that the researchers' heightened awareness - indeed self-criticism - of the limitations of their models, and consequent tinkering with the heterogeneous network that holds them together at all, means that in this field the resources mobilised in the fact-making process themselves remain pointedly visible, resulting in knowledge that is perpetually qualified and situated, as opposed to transcendent.

The first chapter discusses what the researchers see as causes of the complexity of behaviour, especially the important issue of environmental impacts thereupon. Here, Nelson begins to show us how, despite the discipline being behavioral genetics, researchers in the field are compelled to develop - contra the geneticization thesis - a nuanced view of how conditions shape behaviors. Complexity and environmental interference creates a significant stumbling block, especially for newcomers to the field, who may begin to question the likelihood of ever connecting genes and behaviors in a reliable manner. 
These anxieties result in a certain downgrading of the epistemic pretensions of the field as well as demand for a rhetorical strategy that enables the field to sustain itself into the future. Chapter 2 thus shows how new communal understandings of what behavioral genetics can and can't say are forged through narratives of complexity. Past failures and controversies in the field are used as lessons on how to qualify knowledge claims, and to present a view of the field as cautious and a long way from providing knowledge capable of practical application despite its apparent orientation towards immediate and obvious social problems like substance addiction.

Chapter 3 takes the reader deep into the epistemological core of the field by examining in detail what kinds of knowledge researchers feel animal models actually authorise. In particular, it examines how complexity results in the foregrounding of various underlying or infrastructural operations connected to model validation, but which tend to go unnoticed in studies focused on the production of facts. Taking this further, Chapter 4 looks at the other knowledges - beyond or prior to facts - that laboratory practice constructs. Here again the focus is on the generation of knowledge about environmental constraints to genetic causation, and the use of this knowledge in managing claims made in the field. Chapter 5 takes up a specific example - research on binge drinking - to drive home the point that the field is not straightforwardly reductionistic: the requirements of performing the research has actually revealed pathways for thinking about substance abuse as structurally as well as genetically conditioned. Chapter 6 closes out the discussion by opening it up, and examining how researchers cope with having to take their claims public and engage with the media. Here, their now-habitual cautiousness does not play well, and they are incentivised to make bolder statements, and researchers begin to lose control of the resources they've cultivated for describing the relationship between experiments on animals and human-oriented knowledge.

Along the way, Nelson coins and develops a number of useful metaphors and concepts, including "extrafactual", "epistemic by product" and "epistemic scaffold", all intended to aid characterisation of the active, often arduous, recursive and typically methodologicallyoriented activities that distinguish knowledge claims in this branch of animal research - but which are not headline acts in the matter of fact making. By demonstrating the epistemic relevance of these so well, it may be that Nelson calls herself out by erasing the very distinction upon which her claim to be interested in the "extrafactual" order is based, and which lends the focus of her book novelty. However, this would be a somewhat ungenerous criticism.

Overall, the book is a first-class piece of scholarship: the arguments are clearly made, stimulating and well-supported. It contributes significantly to continued interest in the centrality of model organisms to the socio-epistemic character of the modern biosciences. Its excellence has already been recognised, Model Behaviour winning a First Book Award from the UW-Madison Center for the Humanities. It will interest those working on all aspects of social genomics, those who study laboratory animal research and modelling and, because of its re-entry into the constructionist heartland of STS itself, a wide range of scholars concerned with the development of the discipline more widely. 